\title{
Angiographic functional scoring of coronary artery disease predicts mortality in patients with severe aortic stenosis undergoing TAVR
}

Michael Michail $^{\text {ab }}$

Udit Thakur $^{\mathrm{a}}$

Andrea Comella ${ }^{\mathrm{a}}$

Ren Y. Lim ${ }^{\mathrm{a}}$

Vivek Gupta ${ }^{\mathrm{a}}$

Sean $\operatorname{Tan}^{\mathrm{a}}$

Hashrul Rashid ${ }^{\mathrm{a}}$

James D. Cameron ${ }^{\mathrm{a}}$

Stephen J. Nicholls ${ }^{\mathrm{a}}$

Liam M. McCormick ${ }^{\mathrm{a}}$

Robert P. Gooley

Anthony Mathur ${ }^{\mathrm{bc}}$

Alun D. Hughes ${ }^{b}$

Adam J. Brown ${ }^{\mathrm{a}}$

${ }^{a}$ Monash Cardiovascular Research Centre, Monash University, MonashHeart, Monash Health, Melbourne, Australia

${ }^{\mathrm{b}}$ Institute of Cardiovascular Science, University College London, London, United Kingdom

${ }^{\mathrm{c}}$ Centre for Cardiovascular Medicine and Device Innovation, Queen Mary University of London, London, United Kingdom 


\begin{abstract}
Background/purpose Coronary artery disease (CAD) is common in patients undergoing transcatheter aortic valve replacement (TAVR), although its prognostic significance is questionable. Significant CAD stratified using SYNTAX score (SS) has been associated with greater mortality, yet it is unknown whether the functional impact of CAD also impacts outcomes in this cohort. DILEMMA score (DS) is a validated angiographic functional scoring tool that correlates with fractional flow reserve and instantaneous wave-free ratio.
\end{abstract}

This study sought to assess the functional impact of CAD on outcomes in patients undergoing TAVR for severe aortic stenosis (AS).

Methods/materials 229 patients were included in this analysis. Patients underwent angiographic DS and SS and were classified using predefined values. The primary endpoint was one-year all-cause mortality, with secondary endpoints of 30-day major adverse cardiac and cerebrovascular events (MACCE).

Results The mean age was $83.9 \pm 0.5$ years $(55.0 \%$ female), with $11.8 \%$ all-cause mortality. CAD defined by $\geq 30 \%$ stenosis in any vessel was not associated with adverse outcomes $(\mathrm{HR}=1.08, \mathrm{p}=0.84)$. However, the risk of one-year mortality was greater in patients with either $\mathrm{SS}>9(20.8 \%$ vs. $9.4 \%, \mathrm{HR} 2.34, \mathrm{p}=0.03)$ or $\mathrm{DS}>2(18.4 \%$ vs. $8.5 \%, \mathrm{HR}=2.28$, $\mathrm{p}=0.03$ ). Both scoring systems were also associated with 30-day MACCE (both $\mathrm{p}<0.05$ ). After multivariate adjustment, independent predictors of one-year mortality were DS $>2$ $(\mathrm{HR}=2.29, \mathrm{p}=0.04)$, left ventricular ejection fraction $<50 \%(\mathrm{HR} 2.66, \mathrm{p}=0.04)$ and COPD (HR 2.43, $\mathrm{p}=0.04)$.

Conclusion Our results demonstrate that angiographic functional scoring is independently predictive of both 12-month mortality and 30-day MACCE following TAVR.

Keywords: Transcatheter aortic valve replacement, Aortic stenosis, coronary artery disease, Coronary physiology 
Abbreviations

\begin{tabular}{|l|l|}
\hline AS & aortic stenosis \\
\hline BARI MJI & $\begin{array}{l}\text { Bypass Angioplasty Revascularization } \\
\text { Investigation Myocardial Jeopardy Index }\end{array}$ \\
\hline CAD & coronary artery disease \\
\hline CFR & coronary flow reserve \\
\hline CVA & cerebrovascular accident \\
\hline DS & DILEMMA score \\
\hline FFR & fractional flow reserve \\
\hline HR & hazard ratio \\
\hline LVEF & left ventricular ejection fraction \\
\hline MI & myocardial infarction \\
\hline PCI & percutaneous coronary intervention \\
\hline PPMI & periprocedural myocardial injury \\
\hline SAVR & surgical aortic valve replacement \\
\hline SS & SYNTAX score \\
\hline TAVR & transcatheter aortic valve replacement \\
\hline
\end{tabular}




\section{Introduction}

The evolution of transcatheter aortic valve replacement (TAVR) continues to revolutionize the treatment of patients with severe aortic stenosis (AS). However, the management of concomitant coronary artery disease (CAD) remains controversial. In patients undergoing surgical aortic valve replacement (SAVR), revascularization of significant CAD with coronary artery bypass surgery $(\mathrm{CABG})$ reduces the risk of adverse procedural outcomes [1] and is guideline-recommended [2]. It is therefore of widespread interest whether these same principles should be extrapolated to patients undergoing transcatheter therapies.

The influence of CAD on TAVR outcomes has previously been explored in large registries with conflicting results [[3], [4], [5], [6]]. Given the heterogenous nature of CAD, angiographic complexity using SYNTAX scoring (SS) was explored as an alternative means to stratify outcomes [[7], [8], [9], [10]]. A pooled analysis of 3107 patients demonstrated that those with higher residual SS were at greater risk of subsequent mortality [11]. Although the mechanisms are unclear, the presence of significant CAD has been associated with periprocedural myocardial injury (PPMI) [12], with PPMI predictive of both 30-day and 1year mortality [13]. Whilst SS provides an overall assessment of the extent of CAD, it does not assess the prognostic significance of lesion-level or vessel-specific ischemia.

The DILEMMA score (DS) is a validated angiographic scoring tool that strongly correlates with fractional flow reserve (FFR) and instantaneous wave-free ratio (iFR) in patients with stable angina and acute coronary syndromes [[14], [15], [16]]. DS incorporates minimal luminal diameter, lesion length and Bypass Angioplasty Revascularization Investigation Myocardial Jeopardy Index (reflecting the percentage of myocardium subtended by a lesion). It provides a score ranging from 0 to 12 , with a score $\leq 2$ having an excellent negative predictive value for identifying lesions with FFR $>0.80$ and iFR $>0.89$.

In this study we sought to assess the prognostic impact of CAD on all-cause mortality following TAVR, stratified by angiographic scoring systems that act as surrogates for anatomical complexity (SS) or lesion physiological significance (DS). Our aim to the try and ascertain whether either metric was more predictive on TAVR outcomes.

\section{Materials and methods}

We retrospectively screened 320 patients who underwent TAVR for severe AS between November 2008 and October 2016 at MonashHeart, Melbourne. Patients were deemed as having severe AS on echocardiography and met criteria for TAVR as per consensus Heart Team decision. All patients underwent mandated coronary angiography prior to TAVR. 
Amongst patients with significant CAD, revascularization was undertaken in accordance with Heart Team recommendations, either prior to, or at the time of TAVR. In those patients, the post-revascularization angiograms with residual CAD were used for analysis. Patients were treated with either the Lotus Valve System (Boston Scientific, MA, USA), Medtronic CoreValve or Medtronic Evolut R prostheses (Medtronic, MN, USA). Patients were excluded from analysis if they had [1] prior CABG, [2] tandem coronary stenoses, []ㅡ coronary revascularization during TAVR procedure, or [4] TAVR performed via non-femoral vascular access.

\subsection{Angiographic assessment for CAD}

All angiographic analyses were performed by two experienced readers. Patients with $\geq 30 \%$ angiographic stenosis in at least one artery were considered to have CAD warranting further assessment with DS and SS. SS I scores were calculated using the web-based calculator [17]. Previous studies using SS in patients undergoing TAVR have identified that the majority of patients have a low SS [11], thus making the conventional thresholds (low SS $\leq 22$, intermediate SS 23-32 and high SS $\geq 33$ ) unsuitable for stratification. In order to address this, we defined the threshold as the median SS from all the patients with CAD of $\geq 30 \%$ stenosis on visual assessment. DS were calculated by a separate experienced cardiologist blinded to clinical outcomes and SS results. The full methods have been previously detailed [15]. In short, quantitative coronary angiographic analysis using QAngio (Medis Medical Imaging System BV, Leiden, the Netherlands) was performed to derive minimum luminal diameter (MLD) and lesion length (LL) for each lesion. Bypass Angioplasty Revascularization Investigation Myocardial Jeopardy Index (BARI MJI; percentage of left ventricular myocardium subtended by a lesion) was derived by assigning an index to all vessels based on length and calibre and dividing the sum of vessel scores distal to the culprit lesion by the sum of all vessel scores. The values for MLD, LL and BARI MJI each receive a score, the total of which represents DS (between 0 and 12).

Patients were considered to have non-functionally significant disease if lesions had a DS $\leq 2$ as per previous validation studies $[\underline{14}, \underline{15}]$. Patients were classified into two groups: those with functionally significant CAD as evidenced by DS $>2$ in any of the three epicardial vessels or those with non-functionally significant $\mathrm{CAD}$ as defined by $\mathrm{DS} \leq 2$.

\subsection{Study endpoints and follow up}


The primary endpoint for this study was one-year all-cause mortality. Secondary clinical endpoints were 30-day major adverse cardiac and cerebrovascular events (MACCE), comprising spontaneous MI, stroke, TIA, heart failure-related admissions and all-cause death. Outcomes were defined according to the standardized definitions of the Valve Academic Research Consortium-2 consensus (VARC-2) criteria [18].

\subsection{Statistical analysis}

Data was analyzed using Stata (Stata Corp LP, College Station, TX). Baseline and procedural characteristics were compared according to CAD severity stratified by DS. Continuous variables are presented as mean $\pm \mathrm{SD}$ or median \pm interquartile range (IQR) according to their distribution. Categorical variables are presented as absolute numbers and percentages. Comparisons were made using $t$-tests used for continuous variables and $\chi 2$ tests for unpaired categorical variables. The probabilities of death stratified according to CAD severity by DS and SS were plotted using the Kaplan-Meier method. Univariate and multivariate analyses were performed, the latter using a Cox proportional hazards regression model created with variables from univariate analysis with $p$ value $\leq 0.2$. The proportional hazards assumption for these was satisfied through assessment of both Schoenfeld residuals and log-minus-log survival plots. Hazard ratios (HR) or odds ratios (OR) were reported with $95 \%$ confidence intervals, as appropriate. A two-tailed $\mathrm{p}<0.05$ was considered statistically significant.

\section{Results}

A total of 229 patients were included in the final analysis (Fig. 1). Of those, 123 patients $(53.7 \%)$ had at least $\geq 30 \%$ stenosis in one or more of the vessels warranting further SS and DS assessment. Stratified by DS, 153 patients (66.8\%) had all vessels with DS $\leq 2$ whilst 76 $(33.2 \%)$ had at least one vessel with DS $>2$. Conventional SS thresholds demonstrated that the majority of patients $(98.3 \%)$ had a low (0-22), $1.7 \%$ had intermediate (22-32) and none had high ( $\geq 33$ ) SS. The median SS (excluding all patients with SS of 0 ) was 9 and this was used as the threshold for analysis. Of the 229 patients, 181 patients $(79.0 \%)$ had SS $\leq 9$ and 48 patients $(21.0 \%)$ had SS $>9$. Scheduled follow-up was completed in all patients.

The mean age was $83.9 \pm 0.5$ years and $55.0 \%$ were female. There were lower rates of previous MI $(3.3 \%$ vs $10.5 \%, \mathrm{p}=0.025)$ and lower rates of previous percutaneous coronary intervention (PCI; $11.1 \%$ vs $27.6 \%, \mathrm{p}=0.002$ ) when comparing the $\mathrm{DS} \leq 2$ vs $\mathrm{DS}>2$ groups. 
The remainder of the baseline and procedural characteristics remains comparable between groups. The other baseline and procedural characteristics are summarized in Table 1 , Table 2 .

\subsection{Primary and secondary endpoints}

At 365 days post-TAVR, all-cause mortality was $11.8 \%$. The Kaplan Meier curves demonstrating the differences in mortality stratified by the presence of CAD (defined $\geq 30 \%$ visual stenosis severity in any vessel), DS and SS are presented in Fig. 2. Patients with CAD $\geq 30 \%$ visual stenosis severity in any vessel (not further quantified through angiographic scoring) did not have an increased risk of death (hazard ratio $[\mathrm{HR}]=1.08$, CI 0.51-2.31, $\mathrm{p}=0.84$; Fig. 2A). However, when stratifying using DS or SS, patients with significant CAD had greater mortality. After 365 days, DS >2 was associated with a significantly increased risk of death compared with DS $\leq 2(18.4 \%$ vs. $8.5 \%, \mathrm{HR}=2.28$, CI $1.07-4.84 \mathrm{p}=0.03$; Fig. 2B). Similarly, a $S S>9$ was associated with a significantly increased risk of death compared with a SS $\leq 9$ (20.8\% vs 9.4\%, HR 2.34, CI 1.07-5.11, p = 0.03; Fig. 2C). Utilizing a multivariate Cox proportional hazards regression model, DS >2 maintained a significant effect on survival after adjusting for age, BMI, gender, previous PCI, LVEF $<50 \%$ and COPD (adjusted HR 2.29, CI 1.05-4.99 p = 0.04). After multivariate adjustment using the same variables, SS >9 was no longer predictive of mortality (adjusted HR 1.87, CI 0.82-4.22, $\mathrm{p}=0.13)$.

The post-procedural 30-day outcomes are summarized in Table 3. At 30 days, the unadjusted risk for MACCE was greater for patients whether stratified by DS >2 (OR 2.1, p = 0.03) or by $\mathrm{SS}>9$ (OR 2.8, $\mathrm{p}=0.01)$. Individually, the unadjusted risk for the clinical endpoints of spontaneous MI, stroke, TIA and death were not greater at 30 days when stratified by either SS $>9$ or DS $>2$. However, heart failure admissions at 30 days were greater in patients with SS $>9$ (OR 4.50, CI 1.54-12.9, $\mathrm{p}<0.001)$.

\subsection{Other predictors of mortality}

The univariate analysis for the covariates associated with adverse outcomes is presented in Table 4 and Fig. 3. Neither diabetes (HR 0.73, CI 0.25-2.1, p = 0.56) nor hypertension (HR 0.94, CI 0.41-2.15, $\mathrm{p}=0.89$ ) demonstrated any association with death. There was a trend to increased mortality in patients with LVEF <50\% (HR 2.34, CI 0.95-5.81, p = 0.07) and COPD $(\mathrm{HR}=2.14, \mathrm{CI} 0.98-4.68, \mathrm{p}=0.06)$ although these did not reach statistical significance. Along with DS >2, both LVEF <50\% (HR 2.66, CI 1.03-6.72, $p=0.04$ ) and 
COPD (HR 2.43, CI 1.04-5.68, $\mathrm{p}=0.04$ ) were found to be predictors of mortality following TAVR after multivariate adjustment. Additionally, within the subgroup of patients with LVEF $>50 \%$, those with DS $>2$ were still at increased risk of mortality (HR $=2.75$, CI $1.16-$ $6.54, \mathrm{p}=0.02$ ). These findings suggest that mortality was not being driven only by patients with impaired left ventricular function. The subgroup analysis showing the effect of DS $>2$ and SS $>9$ on the individual covariates is presented in Table S1 in the Data Supplement.

\section{Discussion}

The findings from this study demonstrate that patients undergoing TAVR with CAD stratified by DS $>2$ or SS $>9$ were at greater risk of subsequent mortality. DS $>2$ remained an independent predictor of mortality even after adjustment for other variables known to predict outcome. Our study also demonstrates that patients stratified by both DS $>2$ or SS $>9$ are at higher risk of adverse outcomes as early as 30 days. These results therefore suggest that in patients undergoing TAVR, not only is the angiographic severity of CAD prognostically important, but also the functional significance of lesions. These findings importantly highlight that, similar to non-AS patients, lesions most likely to be ischemia-inducing are prognostically significant.

Whilst SS quantifies the extent and severity of CAD, it provides no information on the functional impact of the disease upon the myocardium it subtends. In patients without AS there is ample data to demonstrate that patients with large burden of ischaemia have worsened prognosis $[\underline{19}, \underline{20]}$. Furthermore, functional testing has a crucial prognostic significance in determining whether revascularization is warranted [21, 22]. As such, there has been a paradigm shift from anatomical to functional CAD assessment when making such decisions. This is now superior to angiographic-guided revascularization and is widely acknowledged as gold standard in clinical guidelines [23].

In patients with severe AS, there are a number of pathophysiological changes that result in greater ischemic potential and impaired coronary flow reserve (CFR), even in the absence of CAD $[\underline{24}, 25]$. This includes the evolution of left ventricular hypertrophy in response to increased afterload, which increases resting myocardial oxygen demand. This is met with upregulation of resting coronary blood flow, mediated by decreased microcirculatory resistance. The ability to further increment coronary blood flow in response to additional demands (such as during exertion) is diminished by abnormal ventriculo-aortic physiology. This cascade of events makes patients with severe AS even more susceptible to ischemia than non-AS patients. The presence of functionally significant CAD in this context further 
increases myocardial ischemic potential. This places these patients at greater risk of mismatch between oxygen supply and demand at times of physiological stress (type II myocardial infarction), which leads to myocardial necrosis. Various physiological stressors occur during TAVR procedures and can place patients at higher risk of such myocardial ischemic injury. This, for example, includes periods of hypotension during rapid pacing, valve positioning and deployment [26]. Given the known association between PPMI and adverse outcomes [13], it is therefore conceivable that this is one of the principle underlying mechanisms for poorer outcomes in those with higher DS undergoing TAVR as demonstrated herein. This is supported by data which has also shown that the presence of CAD increases the likelihood for PPMI and subsequent mortality in patients undergoing TAVR [12]. This data suggests that the functional assessment of CAD allows the risk stratification of patients prior to TAVR. Consequently, it raises an important question as to whether revascularisation of functionally significant disease may decrease the risk of adverse events. There are several studies that have demonstrated the feasibility and safety of pressure wire assessment in patients with severe AS [27,28]. Whilst these are promising, there is ongoing debate regarding the most appropriate pressure index in such circumstances [[28], [29], [30]]. Several factors need to be considered including the safety of hyperemic agents in severe AS versus use of non-hyperemic pressure ratios. Additionally, there may be advantages to using a diastolic-only pressure ratio which may be better suited to the altered physiology [30]. Beyond the validation of these techniques, further research is warranted to investigate whether these technologies can better refine the selection of lesions for upfront revascularization. The NOTION-3 (CT03058627) and FAITAVI (CT03360591) randomized trials are currently underway to investigate the outcomes of upstream FFRguided PCI in patients undergoing TAVR.

\subsection{Study limitations}

This is a single-center, observational study and whilst we performed multivariate analyses to account for potential confounders, these results will be subject to other unmeasured effects. Despite endeavours to ensure a completed database, there were some missing data items resulting in the exclusion of patients. Additionally, angiographic scoring tools such as the SS and DS are manually assessed. Nonetheless, in previous validation studies DS demonstrated excellent intra- and inter-operator reproducibility. Whilst SS can be susceptible to variability amongst inexperienced users, these issues were mitigated by using experienced cardiologists who were blinded to the outcomes. Finally, the DS is ultimately a surrogate for FFR and neither are validated in patients with severe AS. As such, further research is required to 
validate the appropriate conditions and thresholds for invasive pressure indices in this patient cohort.

\section{Conclusions}

Angiographic scoring tools that assess anatomical complexity and lesion physiological significance predict clinical outcomes following TAVR. However, only angiographic functional scoring remained independently associated when adjusted for other clinical factors. Further research is now warranted to validate the use of invasive and non-invasive pressure-based indices in severe AS and their role in guiding upstream revascularisation prior to TAVR. 


\section{Funding}

AJB is supported by fellowships from the National Health and Medical Research Council and the National Heart Foundation.

\section{CRediT authorship contribution statement}

Michael Michail: Conceptualization, Methodology, Writing - original draft.

Udit Thakur: Formal analysis, Visualization.

Andrea Comella: Formal analysis.

Ren Y. Lim: Investigation.

Vivek Gupta: Investigation.

Sean Tan: Investigation.

Hashrul Rashid: Data curation, Validation.

James D. Cameron: Supervision, Resources.

Stephen J. Nicholls: Supervision.

Liam M. McCormick: Investigation.

Robert P. Gooley: Investigation.

Anthony Mathur: Supervision.

Alun D. Hughes: Supervision.

Adam J. Brown: Conceptualization, Methodology, Writing - review \& editing.

\section{Declaration of competing interest}

The authors have no conflicts of interest to declare. 


\section{References}

[1] C.J. Mullany, L.R. Elveback, R.L. Frye, J.R. Pluth, W.D. Edwards, T.A. Orszulak, et al. Coronary artery disease and its management: influence on survival in patients undergoing aortic valve replacement J Am Coll Cardiol, 10 (1) (1987), pp. 66-72

[2] H. Baumgartner, V. Falk, J.J. Bax, M. De Bonis, C. Hamm, P.J. Holm, et al. 2017 ESC/EACTS Guidelines for the management of valvular heart disease: the Task Force for the Management of Valvular Heart Disease of the European Society of Cardiology (ESC) and the European Association for Cardio-Thoracic Surgery (EACTS) Eur Heart J, 38 (36) (2017), pp. 2739-2791

[3] P.F. Ludman, N. Moat, M.A. de Belder, D.J. Blackman, A. Duncan, W. Banya, et al. Transcatheter aortic valve implantation in the United Kingdom: temporal trends, predictors of outcome, and 6-year follow-up: a report from the UK Transcatheter Aortic Valve Implantation (TAVI) Registry, 2007 to 2012 Circulation, 131 (13) (2015), pp. 1181-1190

[4] V. Singh, A.P. Rodriguez, B. Thakkar, N.J. Patel, A. Ghatak, A.O. Badheka, et al. Comparison of outcomes of transcatheter aortic valve replacement plus percutaneous coronary intervention versus transcatheter aortic valve replacement alone in the United States Am J Cardiol, 118 (11) (2016), pp. 1698-1704

[5] A. Linke, P. Wenaweser, U. Gerckens, C. Tamburino, J. Bosmans, S. Bleiziffer, et al. Treatment of aortic stenosis with a self-expanding transcatheter valve: the International Multi-centre ADVANCE Study Eur Heart J, 35 (38) (2014), pp. 2672-2684

[6] G.P. Ussia, M. Barbanti, A. Colombo, G. Tarantini, A.S. Petronio, F. Ettori, et al.Impact of coronary artery disease in elderly patients undergoing transcatheter aortic valve implantation: insight from the Italian CoreValve Registry Int J Cardiol, 167 (3) (2013), pp. $943-950$

[7] M.Z. Khawaja, K.N. Asrress, H. Haran, S. Arri, I. Nadra, K. Bolter, et al.The effect of coronary artery disease defined by quantitative coronary angiography and SYNTAX score upon outcome after transcatheter aortic valve implantation (TAVI) using the Edwards bioprosthesis EuroIntervention, 11 (4) (2015), pp. 450-455

[8] G. Witberg, E. Regev, S. Chen, A. Assali, I.M. Barbash, D. Planer, et al.The prognostic effects of coronary disease severity and completeness of revascularization on mortality in patients undergoing transcatheter aortic valve replacement JACC Cardiovasc Interv, 10 (14) (2017), pp. 1428-1435

[9] G.G. Stefanini, S. Stortecky, D. Cao, J. Rat-Wirtzler, C.J. O'Sullivan, S. Gloekler, et al. Coronary artery disease severity and aortic stenosis: clinical outcomes according to SYNTAX score in patients undergoing transcatheter aortic valve implantation Eur Heart J, 35 (37) (2014), pp. 2530-2540

[10] J.M. Paradis, J.M. White, P. Genereux, M. Urena, D. Doshi, T. Nazif, et al.Impact of coronary artery disease severity assessed with the SYNTAX score on outcomes following transcatheter aortic valve replacement J Am Heart Assoc (2017), p. 6(2) 
[11] G. Witberg, O. Zusman, P. Codner, A. Assali, R. KornowskiImpact of coronary artery revascularization completeness on outcomes of patients with coronary artery disease undergoing transcatheter aortic valve replacement. A meta-analysis of studies using the residual SYNTAX score (synergy between PCI with taxus and cardiac surgery) 11 (3) (2018)

[12] K.C. Koskinas, S. Stortecky, A. Franzone, C.J. O’Sullivan, F. Praz, K. Zuk, et al.Postprocedural troponin elevation and clinical outcomes following transcatheter aortic valve implantation J Am Heart Assoc (2016), p. 5(2)

[13] M. Michail, N. Cameron James, N. Nerlekar, R. Ihdayhid Abdul, M. McCormick Liam, R. Gooley, et al.Periprocedural myocardial injury predicts short- and long-term mortality in patients undergoing transcatheter aortic valve replacement Circ Cardiovasc Interv, 11 (11) (2018), Article e007106

[14] M. Michail, H.M. Dehbi, N. Nerlekar, J.E. Davies, A.S.P. Sharp, S. Talwar, et al.Application of the DILEMMA score to improve lesion selection for invasive physiological assessment Catheter Cardiovasc Interv, 94 (2019), pp. E96-E103

[15] D.T. Wong, O. Narayan, B.S. Ko, D.P. Leong, S. Seneviratne, E.L. Potter, et al.A novel coronary angiography index (DILEMMA score) for prediction of functionally significant coronary artery stenoses assessed by fractional flow reserve: a novel coronary angiography index Am Heart J, 169 (4) (2015), pp. 564-571

[16] O. Beton, H. Kaya, O.O. Turgut, M.B. YilmazPrediction of fractional flow reserve with angiographic DILEMMA score Anatol J Cardiol, 17 (4) (2017), pp. 285-292

[17] G. Sianos, M.A. Morel, A.P. Kappetein, M.C. Morice, A. Colombo, K. Dawkins, et al.The SYNTAX Score: an angiographic tool grading the complexity of coronary artery disease EuroIntervention, 1 (2) (2005), pp. 219-227

[18] A.P. Kappetein, S.J. Head, P. Genereux, N. Piazza, N.M. van Mieghem, E.H. Blackstone, et al.Updated standardized endpoint definitions for transcatheter aortic valve implantation: the Valve Academic Research Consortium- 2 consensus document (VARC-2) Eur J Cardiothorac Surg, 42 (5) (2012), pp. S45-S60

[19] R. Hachamovitch, S.W. Hayes, J.D. Friedman, I. Cohen, D.S. BermanComparison of the short-term survival benefit associated with revascularization compared with medical therapy in patients with no prior coronary artery disease undergoing stress myocardial perfusion single photon emission computed tomography Circulation, 107 (23) (2003), pp. 2900-2907

[20] S. Dorbala, R. Hachamovitch, Z. Curillova, D. Thomas, D. Vangala, R.Y. Kwong, et al. Incremental prognostic value of gated $\mathrm{Rb}-82$ positron emission tomography myocardial perfusion imaging over clinical variables and rest LVEF JACC Cardiovasc Imaging, 2 (7) (2009), pp. 846-854

[21] P.A.L. Tonino, B. De Bruyne, N.H.J. Pijls, U. Siebert, F. Ikeno, M. van't Veer, et al. Fractional flow reserve versus angiography for guiding percutaneous coronary intervention $\mathrm{N}$ Engl J Med, 360 (3) (2009), pp. 213-224 
[22] B. De Bruyne, N.H. Pijls, B. Kalesan, E. Barbato, P.A. Tonino, Z. Piroth, et al. Fractional flow reserve-guided PCI versus medical therapy in stable coronary disease N Engl J Med, 367 (11) (2012), pp. 991-1001

[23] F.-J. Neumann, M. Sousa-Uva, A. Ahlsson, F. Alfonso, A.P. Banning, U. Benedetto, et al. 2018 ESC/EACTS Guidelines on myocardial revascularization Eur Heart J (2018), p. ehy394

[24] M. Michail, J.E. Davies, J.D. Cameron, K.H. Parker, A.J. Brown Pathophysiological coronary and microcirculatory flow alterations in aortic stenosis Nat Rev

Cardiol, 15 (7) (2018), pp. 420-431

[25] M. Michael, et al.Acute effects of transcatheter aortic valve replacement on central aortic hemodynamics in patients with severe aortic stenosis

Hypertension (2020), 10.1161/HYPERTENSIONAHA.119.14385 [Epub ahead of print]

[26] M. Michail, K.N. Asrress, J.D. Cameron, R. Gooley, L.M. McCormick, A.D. Hughes, et al. Adaptations to coronary physiology in a patient with severe aortic stenosis and complete heart block undergoing transcatheter aortic valve replacement JACC Cardiovasc Interv, 12 (7) (2019), pp. 687-689

[27] G. Pesarini, R. Scarsini, C. Zivelonghi, A. Piccoli, A. Gambaro, L. Gottin, et al. Functional assessment of coronary artery disease in patients undergoing transcatheter aortic valve implantation: influence of pressure overload on the evaluation of lesions severity Circ Cardiovasc Interv (2016), p. 9(11)

[28] R. Scarsini, G. Pesarini, C. Zivelonghi, A. Piccoli, V. Ferrero, M. Lunardi, et al. Physiologic evaluation of coronary lesions using instantaneous wave-free ratio (iFR) in patients with severe aortic stenosis undergoing trans-catheter aortic valve implantation EuroIntervention, 13 (13) (2017), pp. 1512-1519

[29] F. Yamanaka, K. Shishido, T. Ochiai, N. Moriyama, K. Yamazaki, A. Sugitani, et al. Instantaneous wave-free ratio for the assessment of intermediate coronary artery stenosis in patients with severe aortic valve stenosis: comparison with myocardial perfusion scintigraphy JACC Cardiovasc Interv, 11 (20) (2018), pp. 2032-2040

[30] Y. Ahmad, M. Gotberg, C. Cook, J.P. Howard, I. Malik, G. Mikhail, et al. Coronary hemodynamics in patients with severe aortic stenosis and coronary artery disease undergoing transcatheter aortic valve replacement: implications for clinical indices of coronary stenosis severity JACC Cardiovasc Interv, 11 (20) (2018), pp. 2019-2031 
Table 1. Baseline patient and echocardiographic characteristics.

$\begin{array}{cccccc}\text { DILEMMA } & \text { DILEMMA } & \mathbf{p} & \text { SYNTAX } & \text { SYNTAX } & p \\ \text { score } \leq 2 & \text { score }>2 & \text { value } & \text { score } \leq 9 & \text { score }>9 & \text { value } \\ (n=153) & (n=76) & & (n=181) & (n=48) & \end{array}$

Baseline patient characteristics

\begin{tabular}{|c|c|c|c|c|c|c|}
\hline Age, years & $83.9 \pm 8.5$ & $83.9 \pm 5.4$ & 0.98 & $83.7 \pm 8.0$ & $84.5 \pm 5.8$ & 0.536 \\
\hline Female gender & $85(55.6)$ & $41(53.9)$ & 0.818 & $106(58.6)$ & $28(41.7)$ & 0.036 \\
\hline BMI & $26.7 \pm 5.6$ & $26.8 \pm 6.1$ & 0.923 & $86.8 \pm 5.9$ & $26.5 \pm 5.4$ & 0.731 \\
\hline Diabetes & $28(18.3)$ & $15(19.7)$ & 0.793 & $34(18.8)$ & $9(18.8)$ & 0.996 \\
\hline HTN & $106(69.3)$ & $57(75.0)$ & 0.368 & $131(72.4)$ & $32(66.7)$ & 0.438 \\
\hline $\mathrm{AF}$ & $47(30.7)$ & $25(32.9)$ & 0.738 & $53(29.3)$ & $19(39.6)$ & 0.172 \\
\hline Previous MI & $5(3.3)$ & $8(10.5)$ & 0.025 & $8(4.4)$ & $5(10.4)$ & 0.110 \\
\hline Previous PCI & $17(11.1)$ & $21(27.6)$ & 0.002 & $27(14.9)$ & $11(22.9)$ & 0.185 \\
\hline Previous CVA & $17(11.1)$ & $12(15.8)$ & 0.316 & $24(13.3)$ & $5(10.4)$ & 0.599 \\
\hline COPD & $30(19.6)$ & $21(27.6)$ & 0.169 & $39(21.55)$ & $12(25.0)$ & 0.609 \\
\hline $\begin{array}{l}\text { Creatinine, } \\
\mu \mathrm{mol} / \mathrm{L}\end{array}$ & $96.4 \pm 44.7$ & $96.2 \pm 31.5$ & 0.974 & $95.6 \pm 42.7$ & $99.2 \pm 29.4$ & 0.576 \\
\hline Baseline echocardi & ographic para & & & & & \\
\hline Bicuspid valve & $2(1.3)$ & $1(1.3)$ & 0.996 & $3(1.7)$ & $0(0)$ & 0.996 \\
\hline Valve area, $\mathrm{cm}^{2}$ & $0.74 \pm 0.2$ & $0.7 \pm 0.2$ & 0.245 & $0.74 \pm 0.2$ & $0.68 \pm 0.2$ & 0.068 \\
\hline LVEF $<50 \%$ & $20(13.1)$ & $7(9.2)$ & 0.394 & $18(9.9)$ & $9(18.8)$ & 0.093 \\
\hline $\begin{array}{l}\text { Peak gradient, } \\
\mathrm{mmHg}\end{array}$ & $83.0 \pm 26.0$ & $81.8 \pm 20.9$ & 0.718 & $82.7 \pm 25.0$ & $82.3 \pm 22.2$ & 0.922 \\
\hline $\begin{array}{l}\text { Mean gradient, } \\
\mathrm{mmHg}\end{array}$ & $50.1 \pm 16.3$ & $49.0 \pm 12.3$ & 0.602 & $49.8 .1 \pm 15.6$ & $49.8 \pm 13.1$ & 0.983 \\
\hline $\begin{array}{l}\text { Aortic Velocity, } \\
\mathrm{ms}^{-1}\end{array}$ & $4.4 \pm 0.9$ & $4.5 \pm 0.5$ & 0.485 & $4.5 \pm 0.8$ & $4.5 \pm 0.9$ & 0.683 \\
\hline $\begin{array}{l}\text { Dimensionless } \\
\text { index }\end{array}$ & $0.22 \pm 0.17$ & $0.20 \pm 0.05$ & 0.349 & $0.22 \pm 0.15$ & $0.20 \pm 0.06$ & 0.315 \\
\hline None/mild & $142(92.8)$ & $73(96.1)$ & 002 & $168(92.8)$ & 47 (97.9) & 0 \\
\hline AR Mod/severe & $11(7.2)$ & $3(3.9)$ & 0.335 & $13(7.2)$ & $1(2.1)$ & .190 \\
\hline None/mild & $141(92.2)$ & $67(88.2)$ & & $167(92.3)$ & $41(85.4)$ & \\
\hline Mod/severe & $12(7.8)$ & $9(11.8)$ & 0.3 & $14(7.7)$ & 7 (14.6) & \\
\hline
\end{tabular}


Values are presented as $\mathrm{n}(\%)$ or mean $\pm \mathrm{SD}$. AF indicates atrial fibrillation; AR, aortic regurgitation; BMI, body mass index; COPD, chronic obstructive pulmonary disease; CVA, cerebrovascular accident; LVEF, left ventricular ejection fraction; MI, myocardial infarction; MR, mitral regurgitation; PCI, percutaneous coronary intervention. 
Table 2. Procedural data.

\begin{tabular}{|c|c|c|c|c|c|c|}
\hline & $\begin{array}{c}\text { DILEMMA } \\
\text { score } \leq 2\end{array}$ & $\begin{array}{c}\text { DILEMMA } \\
\text { score }>2\end{array}$ & $\underset{\text { value }}{\mathbf{p}}$ & $\begin{array}{l}\text { SYNTAX } \\
\text { score } \leq 9\end{array}$ & $\begin{array}{l}\text { SYNTAX } \\
\text { score }>9\end{array}$ & $\begin{array}{c}\mathbf{p} \\
\text { value }\end{array}$ \\
\hline Self-expanding valve & $62(40.5)$ & $30(39.5)$ & 0.879 & $72(39.8)$ & $20(41.7)$ & 0.813 \\
\hline Length of hospital stay & $8.6 \pm 6.2$ & $9.0 \pm 7.1$ & 0.651 & $8.9 \pm 6.8$ & $8.3 \pm 5.3$ & 0.605 \\
\hline $\begin{array}{c}\text { VARC } 0 \text { or } \\
1\end{array}$ & $122(79.7)$ & $59(77.6)$ & \multirow[b]{2}{*}{0.712} & $141(77.9)$ & $40(83.3)$ & \multirow[b]{2}{*}{0.411} \\
\hline $\begin{array}{c}\text { Bleeding } \\
\text { VARC } 2 \text { or } \\
\mathbf{3}\end{array}$ & $31(20.3)$ & $17(22.4)$ & & $40(22.1)$ & $8(16.7)$ & \\
\hline Vascular complication & $39(25.5)$ & $18(23.7)$ & 0.766 & $52(28.7)$ & $5(10.4)$ & 0.009 \\
\hline No AKI & $134(87.6)$ & $63(82.9)$ & & $157(86.7)$ & $40(83.3)$ & \\
\hline \multirow{2}{*}{ AKI } & $11(7.2)$ & $7(9.2)$ & 0707 & $14(7.7)$ & $4(8.3)$ & \multirow{3}{*}{0.692} \\
\hline & $4(2.6)$ & $4(5.3)$ & 0.102 & $5(2.8)$ & $3(6.3)$ & \\
\hline Stage 3 & $4(2.6)$ & $2(2.6)$ & & $5(2.8)$ & $1(2.1)$ & \\
\hline LVEF, $<50 \%$ & $16(10.5)$ & $14(18.4)$ & 0.093 & $16(8.8)$ & $14(29.2)$ & $<0.001$ \\
\hline Peak gradient, $\mathrm{mmHg}$ & $22.2 \pm 8.7$ & $20.6 \pm 11.3$ & 0.248 & $22.6 \pm 10.0$ & $18.3 \pm 7.0$ & 0.007 \\
\hline Mean gradient, $\mathrm{mmHg}$ & $11.7 \pm 4.8$ & $10.5 \pm 6.3$ & 0.119 & $11.8 \pm 5.6$ & $9.6 \pm 3.8$ & 0.012 \\
\hline DI & $0.54 \pm 0.16$ & $0.52 \pm 0.16$ & 0.406 & $0.53 \pm 0.17$ & $0.53 \pm 0.13$ & 0.962 \\
\hline None/mild & $149(97.4)$ & $75(98.7)$ & \multirow{2}{*}{0.527} & $177(97.8)$ & 47 (97.9) & \multirow{2}{*}{0.957} \\
\hline Mod/severe & $4(2.6)$ & $1(1.3)$ & & $4(2.2)$ & $1(2.1)$ & \\
\hline \multirow{3}{*}{ Mod/ } & $144(94.1)$ & $71(93.4)$ & \multirow{2}{*}{0.836} & $172(95.0)$ & 43 (89.6) & \multirow{2}{*}{0.162} \\
\hline & $9(5.9)$ & $5(6.6)$ & & $9(5.0)$ & 5 (10.4) & \\
\hline & $36(23.5)$ & $17(22.4)$ & 0.844 & $46(25.4)$ & $12(25.0)$ & 0.953 \\
\hline
\end{tabular}


Table 3. 30-day outcomes.

\begin{tabular}{cllllll} 
& \multicolumn{1}{c}{$\begin{array}{c}\text { DILEMMA } \\
\text { score } \leq \mathbf{2}\end{array}$} & $\begin{array}{c}\text { DILEMMA } \\
\text { score }>\mathbf{2}\end{array}$ & $\begin{array}{c}\mathbf{p} \\
\text { value }\end{array}$ & $\begin{array}{c}\text { SYNTAX } \\
\text { score } \leq \mathbf{9}\end{array}$ & $\begin{array}{c}\text { SYNTAX } \\
\text { score }>\mathbf{9}\end{array}$ & $\begin{array}{c}\mathbf{p} \\
\text { value }\end{array}$ \\
$\begin{array}{c}\text { Spontaneous } \\
\text { MI }\end{array}$ & $3(2.0)$ & $0(0)$ & 0.219 & $3(2.0)$ & $0(0)$ & 0.370 \\
$\begin{array}{c}\text { Stroke and } \\
\text { TIA }\end{array}$ & $6(3.9)$ & $7(9.2)$ & 0.103 & $9(5.0)$ & $4(8.3)$ & 0.371 \\
Death & $3(2.0)$ & $3(4.0)$ & 0.375 & $4(2.2)$ & $2(4.1)$ & 0.451 \\
$\begin{array}{c}\text { HF } \\
\text { admissions }\end{array}$ & $10(6.5)$ & $10(13.2)$ & 0.095 & $10(5.5)$ & $10(20.8)$ & 0.001 \\
MACCE & $21(13.7)$ & $19(25.0)$ & 0.034 & $25(13.8)$ & $15(31.3)$ & 0.005
\end{tabular}

Values are presented as n (\%). Major adverse cardiac and cerebrovascular events (MACCE) included spontaneous myocardial infarction (MI), stroke, transient ischaemic attack (TIA), heart failure (HF) admissions and all-cause death at 30 days. 
Table 4. Univariate analyses.

\begin{tabular}{|c|c|c|c|}
\hline Variable & HR & CI & p value \\
\hline${ }^{\mathrm{a}} \mathrm{Age}$ & 1.044 & $0.97-1.12$ & 0.248 \\
\hline${ }^{\mathrm{b}} \mathrm{BMI}$ & 0.95 & $0.89-1.01$ & 0.105 \\
\hline Male gender & 1.84 & $0.85-3.96$ & 0.120 \\
\hline Self-expanding valve & 0.88 & $0.40-1.92$ & 0.742 \\
\hline Diabetes & 0.73 & $0.25-2.10$ & 0.556 \\
\hline HTN & 0.94 & $0.41-2.15$ & 0.890 \\
\hline Previous CVA & 0.54 & $0.13-2.28$ & 0.402 \\
\hline Previous MI & 2.21 & $0.66-7.33$ & 0.197 \\
\hline Previous PCI & 1.13 & $0.43-2.99$ & 0.800 \\
\hline COPD & 2.14 & $0.98-4.68$ & 0.056 \\
\hline${ }^{\mathrm{c}}$ Creatinine & 1.00 & $0.99-1.01$ & 0.447 \\
\hline LVEF $<50 \%$ & 2.34 & $0.95-5.81$ & 0.066 \\
\hline $\mathrm{DS}>2$ & 2.28 & $1.07-4.84$ & 0.033 \\
\hline $\mathrm{SS}>9$ & 2.34 & $1.07-5.11$ & 0.033 \\
\hline CAD $(\geq 30 \%$ in at least one vessel $)$ & 1.08 & $0.51-2.31$ & 0.840 \\
\hline \multicolumn{4}{|c|}{ Independent predictors of post-transcatheter aortic valve replacement mortality. Hazard ratio } \\
\hline \multicolumn{4}{|c|}{ artery disease; COPD, chronic obstructive pulmonary disease; CVA, cerebrovascular } \\
\hline \multicolumn{4}{|c|}{ accident; DS, DILEMMA score; LVEF, left ventricular ejection fraction; MI, myocardial } \\
\hline infarction; PCI, percutaneous corona & SYNTAY & core. ${ }^{\text {a }}$ Per o & \\
\hline
\end{tabular}




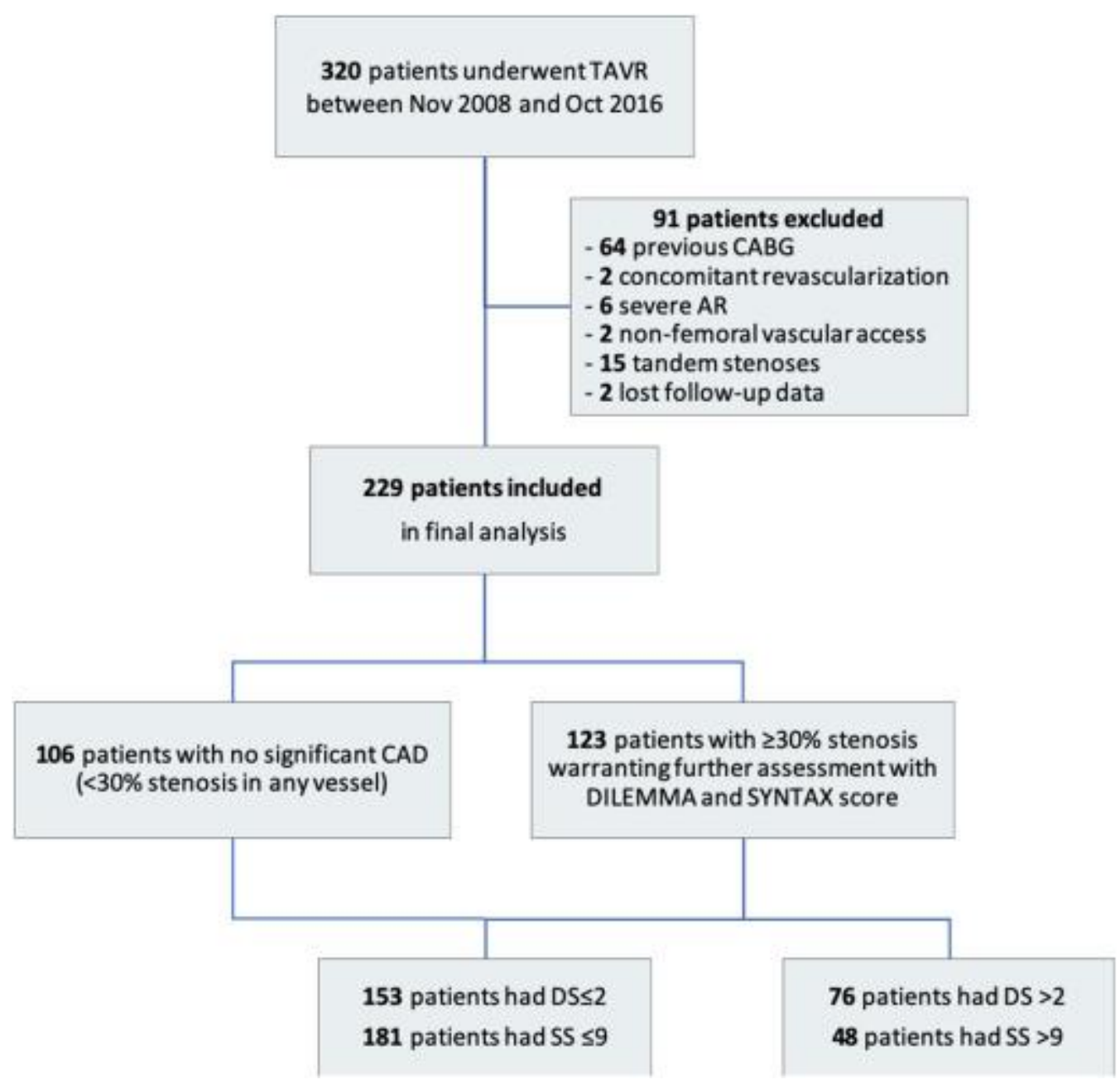

Fig. 1. Study Flow Chart. Of the 320 patients who were screened, 229 patients were included in the final analysis. AR indicates aortic regurgitation; $\mathrm{CABG}$, coronary artery bypass surgery; CAD, coronary artery disease; TAVR, transcatheter aortic valve replacement. 


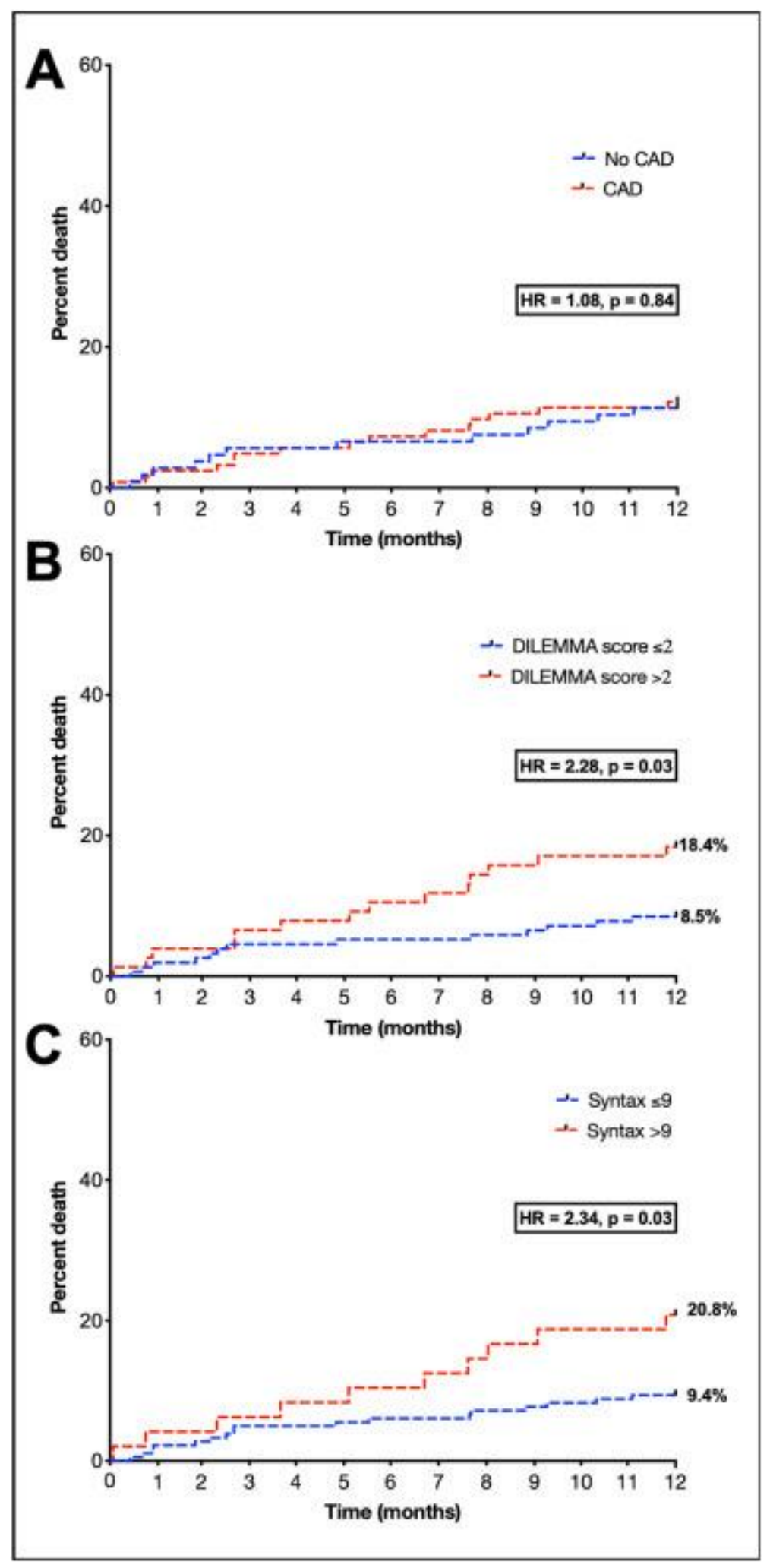

Fig. 2. Kaplan-Meier curves showing the effects of coronary artery disease (CAD) upon post-transcatheter aortic valve replacement mortality as stratified by (A) the presence of CAD defined by $\geq 30 \%$ stenosis in $\geq 1$ vessel, (B) DILEMMA score and (C) SYNTAX score. 


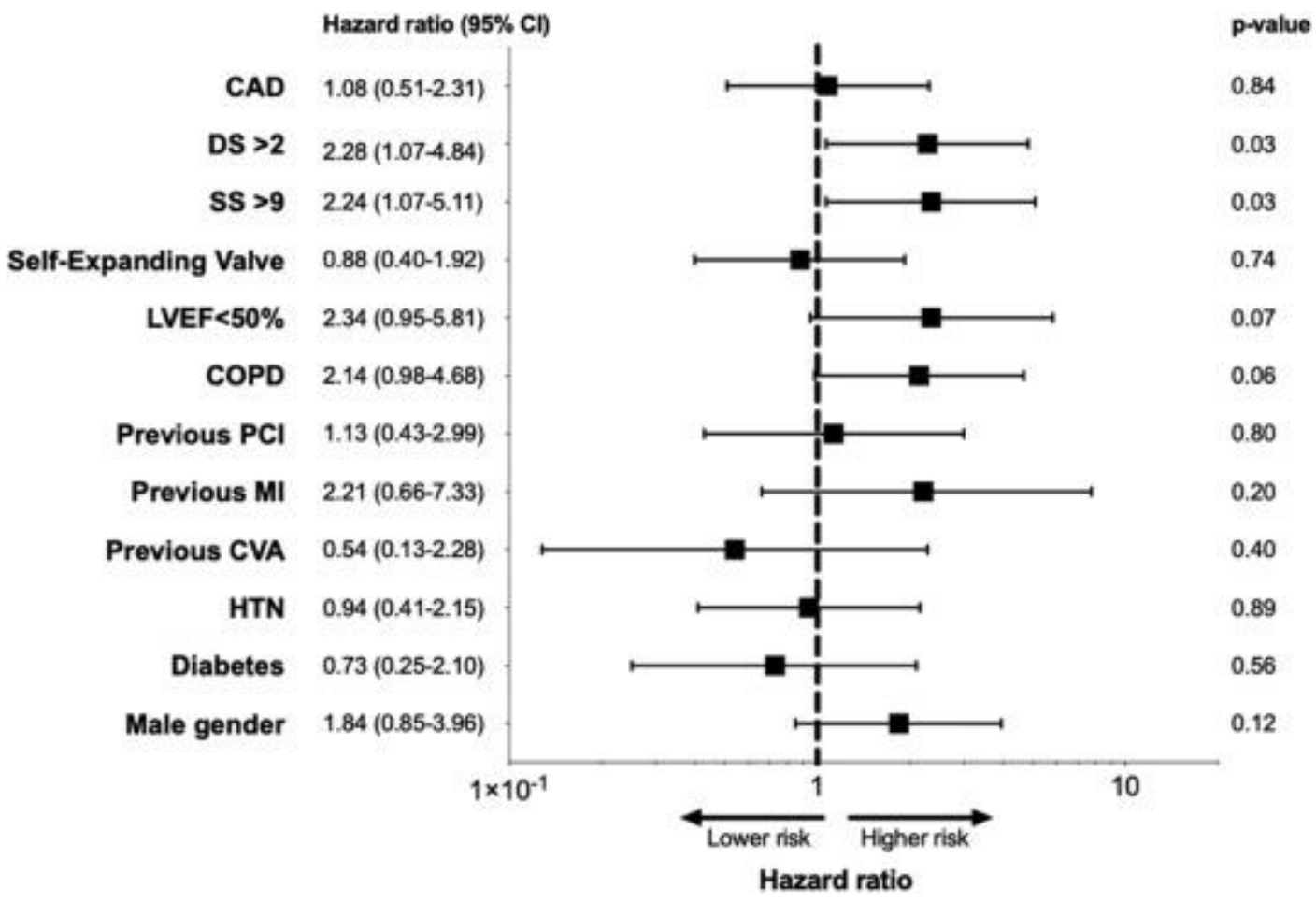

Fig. 3. The assessment of independent predictors of mortality following transcatheter aortic valve replacement on univariate analysis. Left ventricular ejection fraction (LVEF) $<30 \%$ was the only variable identified to have impacted mortality. CAD indicates coronary artery disease ( $\geq 30 \%$ in at least one vessel); CI, confidence intervals; COPD, chronic obstructive pulmonary disease; CVA, cerebrovascular accident; DS, DILEMMA score, HTN, hypertension; MI, myocardial infarction; PCI, percutaneous coronary intervention. 